Eine Umstrukturierung des Energiesystems ist notwendig

\section{Weichenstellungen für eine nachhaltige Stromversorgung}

In der aktuellen Diskussion zur Stromversorgung wird häufig unterstellt, dass das Zieldreieck aus Klimaschutz, Versorgungssicherheit und Wirtschaftlichkeit nur durch einen Mix verschiedener Energieträger erreichbar sei. Die erneuerbaren Energien alleine könnten es nicht schaffen. Doch es gibt gute Gründe, diese Annahme infrage zu stellen.

Von Christian Hey und Olav Hohmeyer

D ie Zukunft der Stromversorgung bis 2050 ist das Thema des nächsten Sondergutachtens des Sachverständigenrates für Umweltfragen. Die zentrale Herausforderung ist dabei der Übergang in eine nachhaltige und zuverlässige Stromerzeugung, die weitestgehend auf Treibhausgasemissionen verzichtet.

\section{Eine Grundsatzentscheidung ist notwendig}

Der Sachverständigenrat für Umweltfragen geht davon aus, dass wir heute vor der Grundsatzentscheidung zwischen zwei sich gegenseitig ausschließenden Entwicklungspfaden für die zukünftige Stromversorgung stehen:

- Möglich ist entweder ein massiver Ausbau der regenerativen Energiequellen, der mit schnell startenden Kraftwerkskapazitäten (Gaskraftwerke), Speichern und einem erheblichen Netzausbau kombiniert werden muss,

I oder der Ausbau einer Kraftwerksstruktur auf der Basis von Grundlastkraftwerken (Kohle mit $\mathrm{CO}_{2}$-Sequestrierung und/oder Kernkraftwerke) unter Verzicht auf einen weiteren substanziellen Ausbau der regenerativen Energiequellen Wind und Sonne zur Stromerzeugung, da ein hoher Anteil von Wind- und Sonnenenergie nicht sinnvoll mit einer grundlastorientierten Stromerzeugung kombiniert werden kann.

Die aktuelle Diskussion um die Zukunft der Stromversorgung in Deutsch- land wird häufig unter der falschen Prämisse geführt, dass Kohlekraftwerke als notwendige Ergänzung der regenerativen Energiequellen für die Bereitstellung von sogenannter Grundlast erforderlich seien. Diese Kohlekraftwerke benötige man insbesondere vor dem Hintergrund des gesetzlich verankerten Ausstiegs aus der Nutzung der Kernenergie. Mit dieser Argumentation wird die energiepolitische Debatte auf die Frage: „Kohle oder Kernenergie?" eingeschränkt.

\section{Das Energiesystem der Zukunft}

Systematische Analysen der Eigenschaften der wichtigsten regenerativen Energiequellen in der Stromerzeugung auf der einen und der Eigenschaften von Grundlastkraftwerken (auf Kohle- oder Kernenergiebasis) auf der anderen Seite zeigen, dass gerade die vergleichsweise kostengünstige und in großem Umfang mögliche Nutzung der Windenergie an Land und auf See nicht mit den technischen und ökonomischen Charakteristika von Grundlastkraftwerken kompatibel ist. Der Anteil der durch konventionelle Grundlastkraftwerke zu deckenden Netzlast würde auf einen Bruchteil der heute installierten Kraftwerksleistung zurückgehen, wenn sich der Ausbau der Windenergie entsprechend der Pläne der Bundesregierung und der Erfordernisse einer kostengünstigen, nachhaltigen langfristigen Elektrizitätsversorgung weiter fortsetzt.
In einem Energiesystem mit hohen Anteilen stark fluktuierender regenerativer Energiequellen verlieren damit Grundlastkraftwerke nicht nur massiv an Bedeutung, sie sind vielmehr aufgrund ihrer technischen Eigenschaften (nur sehr langsames Anfahren über Stunden möglich) nicht mehr geeignet, die notwendigen Aufgaben regelbarer Kraftwerkskapazitäten in dem neuen Energiesystem sinnvoll und kostengünstig zu erfüllen.

Eine Stromversorgung ohne Kohle und Kernenergie auf der Basis regenerativer Energiequellen ist aber nach vielen Potenzialanalysen möglich. Damit lautet die zentrale Frage über die Zukunft der Stromversorgung: ,Stromversorgung auf der Basis von Grundlastkraftwerken (Kohle und/oder Kernenergie) oder auf der Basis regenerativer Energiequellen?"

\section{Der Diskussion eine neue Richtung geben}

Es zeichnet sich dennoch aktuell ab, dass Politik und Energiewirtschaft Investitionsentscheidungen zugunsten einer grundlastorientierten Energieversorgung vorantreiben. Um die dringend erforderliche öffentliche Debatte über die Langfristfolgen dieser die Zukunft der deutschen Energieversorgung prägenden Entscheidungen anzustoßen, hat der Sachverständigenrat für Umweltfragen ein Thesenpapier verfasst, das am 28. Mai 2009 auf einer Konferenz zur Diskussion gestellt wurde.

I These 1: Bis 2050 müssen die Industrieländer ihre Treibhausgasemissionen um mindestens 80 Prozent reduzieren, um einen gefährlichen Klimawandel zu verhindern und eine faire Lastenverteilung zwischen Industrie- und Entwicklungsländern zu ermöglichen.

- These 2: Die Weichenstellungen heute prägen die Emissionen bis 2050. Da viele Kraftwerkstypen eine Lebensdauer von einigen Jahrzehnten haben, muss der Zeithorizont der aktuellen energiepolitischen Diskussion erweitert werden. Investitionsentscheidungen heute dürfen die langfristig not- 
wendige Emissionsreduktion nicht behindern. Es geht nicht mehr nur darum, ob wir unsere klimapolitischen Ziele bis 2020 oder 2030 erreichen, sondern wie wir heute die Grundlagen für einen erfolgreichen Klimaschutz bis 2050 legen können. Die aktuellen Neubaupläne für konventionelle Kohlekraftwerke ohne $\mathrm{CO}_{2}$-Sequestrierung sind daher nicht mit den Klimazielen für 2050 vereinbar.

I These 3: Der Strombedarf in Deutschland und Europa kann weitgehend bis vollständig durch erneuerbare Energien gedeckt werden. Hierfür ist jedoch eine Umstrukturierung des Systems nötig, die insbesondere den Ausbau von Netzen, Leitungen für effiziente Fernübertragung und die Bereitstellung von Speicherkapazitäten einschließt.

Der zeitliche Vorlauf für den Umbau von Netzen und Speicherkapazitäten erfordert bereits frühzeitig Richtungsentscheidungen. Diese Umstrukturierung ist möglich, erfordert jedoch einen starken politischen Gestaltungswillen.

- These 4: Hohe Anteile von Grundlastenergien sind mit dem weiteren Ausbau der erneuerbaren Energien nicht vereinbar. Die Einsatzmöglichkeiten und damit die Wirtschaftlichkeit von Grundlastkraftwerken werden bei einem hohen Anteil erneuerbarer Energien eingeschränkt, stattdessen werden schnellstartende Kraftwerke und Regelenergie gebraucht.

Eine grundlastbasierte Stromerzeugung durch Atom- oder Kohlekraftwerke ist damit nicht mit dem Ausbau erneuerbarer Energien kompatibel; eine Systementscheidung muss getroffen werden. Es ist technisch und ökonomisch nicht sinnvoll, beide Pfade gleichzeitig zu verfolgen.

- These 5: Die Systementscheidung sollte zugunsten der erneuerbaren Energien erfolgen, da die Energieträger Kohle und Kernenergie keine nachhaltige und zukunftsfähige Stromversorgung sicherstellen können. Die Kernenergie ist wegen der ungelösten Endlagerproblematik und weiterer Ri- siken nicht nachhaltig. Das Wissen um die Chancen und Risiken von $\mathrm{CO}_{2}$-Sequestrierung ist derzeit ungenügend. Wegen der begrenzten Speicherkapazitäten für $\mathrm{CO}_{2}$ und begrenzter Uranvorkommen könnten sowohl die Kohleenergie als auch die Atomenergie den Umstieg auf erneuerbare Energien nur für wenige Jahrzehnte aufschieben.

\section{Schlussfolgerungen}

Der Sachverständigenrat für Umweltfragen ist deshalb der Auffassung, dass die derzeitige öffentliche Energiediskussion um die Alternativen Kohle oder Kernenergie in die völlig falsche Richtung führt.

Es ist stattdessen überfällig, die technische, ökonomische und politische Umsetzung einer nachhaltigen und klimaverträglichen Energieversorgung auf der Basis regenerativer Energiequellen auf die Tagesordnung zu heben. Hierbei ist der Zeithorizont mindestens auf das Jahr $2050 \mathrm{zu}$ erweitern.

I AUTOREN + KONTAKT

Dr. Christian Hey ist Generalsekretär des Sachverständigenrates für Umweltfragen. Dr. Olav Hohmeyer ist Professor für Energie- und Ressourcenwirtschaft an der Universität Flensburg und Mitglied im Sachverständigenrat für Umweltfragen.

Sachverständigenrat für Umweltfragen (SRU), Reichpietschufer 60, 10785 Berlin. Tel.: +49 $30263696-0$, Fax:+49 30263696 109, E-Mail:sru-info@uba.de

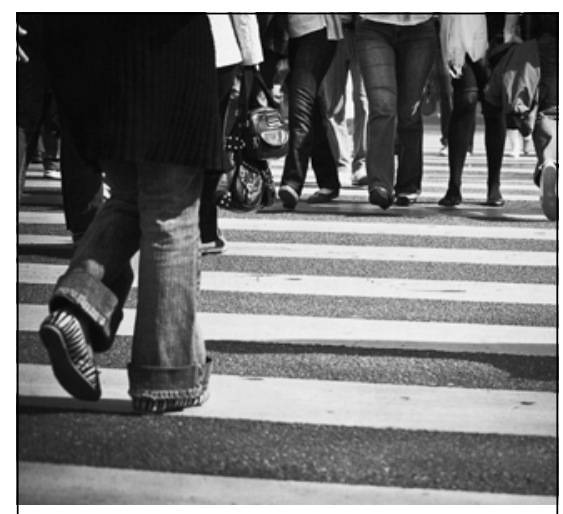

\section{Mobil in die Zukunft}

Eine zentrale Herausforderung für Politik und Wirtschaft ist es, für den Wirtschaftsstandort Deutschland auch in Zukunft ein hohes Maß an Mobilität zu gewährleisten und dabei Klima und Ressourcen zu schonen. Welche Angebote an modernen Mobilitätsdienstleistungen entstehen sollen und was Unternehmen bereits umsetzen, stellt factorY 1/2009 vor - vom Elektroauto über betriebliche Mobilitätsprogramme bis hin zur „Zero-Emission-Mobility“.

factorY $Y_{\text {ist das }}$

Magazin für Nachhaltiges Wirtschaften.

factorY spürt die aktuellen Trends für Nachhaltigkeit in Produktion und Konsum auf.

factorY stellt Unternehmen vor, die in der gesamten Produktionskette nachhaltige Strukturen schaffen.

factorY Probeabo bei factorY Publishing Am Varenholt I23, 44797 Bochum T 0234.9799513, F 0234.9799514 oder www.factory-magazin.de

Einzelheft: 4,6o € zzgl. Versand factorY im Abo: 4 Hefte für 23,50 € Inland, 33,50 € Ausland (inkl. MwSt und Versand)

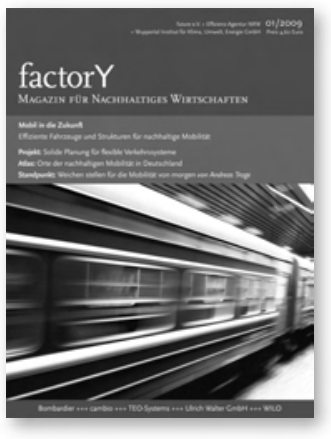


(c) 20I0 Authors; licensee IÖW and oekom verlag. This is an article distributed under the terms of the Creative Commons Attribution Non-Commercial No Derivates License (http://creativecommons.org/licenses/by-nc-nd/3.o/), which permits unrestricted use, distribution, and reproduction in any medium, provided the original work is properly cited. 